Pacific

Journal of

Mathematics

A HODGE DECOMPOSITION FOR THE COMPLEX OF INJECTIVE WORDS

\author{
Phil Hanlon and Patricia Hersh
}

Volume $214 \quad$ No. 1

March 2004 


\title{
A HODGE DECOMPOSITION FOR THE COMPLEX OF INJECTIVE WORDS
}

\author{
Phil Hanlon AND PATRICIA Hersh
}

\begin{abstract}
Reiner and Webb (preprint, 2002) compute the $S_{n}$-module structure for the complex of injective words. This paper refines their formula by providing a Hodge type decomposition. Along the way, this paper proves that the simplicial boundary map interacts in a nice fashion with the Eulerian idempotents.

The Laplacian acting on the top chain group in the complex of injective words is also shown to equal the signed random to random shuffle operator. Uyemura-Reyes, 2002, conjectured that the (unsigned) random to random shuffle operator has integral spectrum. We prove that this conjecture would imply that the Laplacian on (each chain group in) the complex of injective words has integral spectrum.
\end{abstract}

\section{Introduction.}

Let $V=\left\langle v_{1}, \ldots, v_{n}\right\rangle$ be an $n$-dimensional Euclidean space. For each $r$, let $\Gamma_{r}=V^{\otimes r}$ and let $\partial_{r}: \Gamma_{r} \rightarrow \Gamma_{r-1}$ be the map given by:

$$
\partial_{r}\left(a_{1} \otimes \cdots \otimes a_{r}\right)=\sum_{j=1}^{r}(-1)^{j-1}\left(a_{1} \otimes \cdots \otimes a_{j-1} \otimes a_{j+1} \otimes \cdots \otimes a_{r}\right) .
$$

It is well-known that the $\partial_{r}$ are boundary maps, i.e., that $\partial_{r} \cdot \partial_{r+1}=0$.

Let $M_{r}$ be the multilinear part of $\Gamma_{r}$. So, $M_{r}=0$ if $r>n$, and for $r \leq n$,

$$
M_{r}=\left\langle v_{i_{1}} \otimes v_{i_{2}} \otimes \cdots \otimes v_{i_{r}}: i_{1}, \ldots, i_{r} \text { are distinct }\right\rangle .
$$

Note that $\operatorname{dim}\left(M_{r}\right)=n(n-1) \ldots(n-r+1)=\frac{n !}{(n-r) !}$. Also, it is clear that $\partial_{r}\left(M_{r}\right) \subset M_{r-1}$ and so

$$
0 \rightarrow M_{n} \rightarrow M_{n-1} \rightarrow \cdots \rightarrow M_{0} \rightarrow 0
$$

is a subcomplex of $\left(\Gamma_{*}, \partial_{*}\right)$. This paper will concern the homology of this subcomplex.

The complex $\left(M_{*}, \partial_{*}\right)$ appears in earlier work on the subword order of injective words on the alphabet $\{1,2, \ldots, n\}$. This poset is the face poset of a regular CW complex $K_{n}$ whose homology agrees with the homology of $\left(M_{*}, \partial_{*}\right)$. In $[\mathbf{F}]$, Farmer proves that $K_{n}$ is homotopy equivalent to a wedge of $(n-1)$-spheres thus showing that the homology of $\left(M_{*}, \partial_{*}\right)$ vanishes except 
at top degree. Bjorner and Wachs $[\mathbf{B W}]$ prove a stronger result - that the lexicographic order on permutations induces a recursive coatom ordering on the poset of injective words. This in turn gives a dual CL-shelling of $K_{n}$.

Reiner and Webb $[\mathbf{R W}]$ study $\left(M_{*}, \partial_{*}\right)$ as a subcomplex of $\left(\Gamma_{*}, \partial_{*}\right)$. The natural action of $S_{n}$ on $\left\{v_{1}, \ldots, v_{n}\right\}$ extends to an action of $S_{n}$ on $\left(\Gamma_{*}, \partial_{*}\right)$ which preserves $\left(M_{*}, \partial_{*}\right)$. Reiner and Webb compute the homology of $\left(M_{*}\right.$, $\left.\partial_{*}\right)$ as an $S_{n}$-module.

Theorem 1.1 (Reiner-Webb). As an $S_{n}$-module, the top homology of $\left(M_{*}\right.$, $\left.\partial_{*}\right)$ is

$$
\bigoplus_{k=0}^{n}(-1)^{n-k} \text { ind }{ }_{S_{n-k}}^{S_{n}}\left(\varepsilon_{n-k}\right)=\bigoplus_{k=0}^{n}(-1)^{n-k} \text { ind }_{S_{n-k} \times S_{k}}^{S_{n}}\left(\varepsilon_{n-k} \otimes \operatorname{Reg}_{k}\right)
$$

where $\varepsilon_{n-k}$ denotes the trivial representation of $S_{n-k}$. Furthermore, the multiplicity of an irreducible $S^{\lambda}$ of $S_{n}$ in the top homology is equal to the number of standard Young tableaux of shape $\lambda$ which have their smallest descent even.

In this paper, we will do two things. First, we will show that there is a natural Hodge decomposition of the homology of $\left(M_{*}, \partial_{*}\right)$. This decomposition will split $H_{n}(M)$ into $n$ components

$$
H_{n}(M)=\bigoplus_{j=1}^{n} H_{n}^{(j)}(M)
$$

We will show that the dimension of $H_{n}^{(j)}(M)$ is equal to the number of derangements with exactly $j$ cycles. More specifically, we will show that each $H_{n}^{(j)}(M)$ is invariant under the action of $S_{n}$ and prove that $H_{n}^{(j)}(M)$ is a sum of linear characters induced from centralizers of permutations with exactly $j$ cycles.

Second, we will study the Laplacian $\Lambda_{*}$ associated to the complex $\left(M_{*}, \partial_{*}\right)$. We will show that $\Lambda_{n}$ is closely connected to the transition matrix for random to random shuffling. Random to random shuffling has been studied by Uyemura-Reyes in $[\mathbf{U y}]$. In $[\mathbf{U y}]$, the author makes a conjecture about the spectrum of the transition matrix for random to random shuffling which together with our results imply the conjecture that the spectrum of $\Lambda_{n}$ is integral. We go on to compute $\Lambda_{r}$, for $r<n$ in terms of $\Lambda_{n}$. This computation shows that $\Lambda_{r}$ is positive definite for $r<n$ thus giving another proof that $H_{r}(M)=0$ for $0 \leq r<n$. This computation also shows that the spectrum of $\Lambda_{r}$ is integral if the spectrum of $\Lambda_{n}$ is integral. Thus, if Uyemura-Reyes' conjecture on the spectrum of random to random shuffling is correct, then the spectra of all $\Lambda_{r}$ are integral. 


\section{A Hodge type decomposition of $H_{*}(M)$.}

We begin by recalling the definition of the Eulerian idempotents $e_{r}^{(j)}$ in $\mathbf{C} S_{r}$. For each $r$ and $k$, let $S(r ; k)$ denote the set of permutations in $S_{r}$ with exactly $k-1$ descents. Following Loday [Lo], define elements $l_{r}^{(k)}$ and $\lambda_{r}^{(k)}$ in $\mathbf{C} S_{r}$ according to the following formulae:

$$
\begin{aligned}
l_{r}^{(k)} & =(-1)^{k-1} \sum_{\sigma \in S(r ; k)} \operatorname{sgn}(\sigma) \sigma, \\
\lambda_{r}^{(k)} & =\sum_{i=0}^{k-1}(-1)^{i}\left(\begin{array}{c}
n+i \\
i
\end{array}\right) l_{r}^{(k-i)}, \\
(-1)^{k-1} \lambda_{r}^{(k)} & =\sum_{j=1}^{n} k^{j} e_{r}^{(j)} .
\end{aligned}
$$

It is worth noting that the first two equations define the $\lambda_{r}^{(k)}$ explicitly. The third equation then determines the $e_{r}^{(j)}$ in terms of the $\lambda_{r}^{(k)}$ because the transition matrix $\left(k^{j}\right)_{k, j}$ is a Vandermonde and hence invertible.

There is a significant literature on the Eulerian idempotents and their many remarkable properties. We will need two of these properties. The first is the well-known fact that $e_{n}^{(1)}, e_{n}^{(2)}, \ldots, e_{n}^{(n)}$ form a set of pairwise orthogonal idempotents in $\mathbf{C} S_{n}$ that decompose the identity. In other words, $e_{n}^{(j)} \cdot e_{n}^{(\ell)}=0$ if $j \neq \ell$ and $e_{n}^{(1)}+e_{n}^{(2)}+\cdots+e_{n}^{(n)}=i d$. This implies that if $X$ is any $S_{n}$-module, then

$$
X=\bigoplus_{j} e_{n}^{(j)} \cdot X
$$

The second fact we will need describes the relationship between the $e_{n}^{(j)}$ and the boundary map $\partial$. To state this result, it will be helpful to write permutations in one-line notation. Let $i \in\{1,2, \ldots, n\}$ and let $S_{n \backslash\{i\}}$ denote permutations of $\{1,2, \ldots, n\} \backslash\{i\}$. There is a natural identification of $S_{n \backslash\{i\}}$ with $S_{n-1}$ which comes about by changing each occurrence of $i+j$ to $i+j-1$. Via this identification, we can think of $e_{n-1}^{(\ell)}$ as sitting inside of the group algebra of $S_{n \backslash\{i\}}$.

As in $[\mathbf{R W}]$, we will think of $\partial$ as acting on linear combinations of injective words on the alphabet $\{1,2, \ldots, n\}$. For $i \in\{1,2, \ldots, n\}$, let $\partial[i]$ denote the part of $\partial$ which removes the number $i$. The next theorem presents a surprisingly elegant outcome to the computation of $\partial[i] e_{n}^{(k)}$.

Before stating and proving the result, we will give an example to be sure that the notation is clear. Let $n=3$. In the example that follows, we will use $a, b, c$ in place of $1,2,3$ so as to avoid confusion with coefficients. The 
Eulerian idempotents are given by:

$$
\begin{aligned}
e_{3}^{(1)} & =\frac{1}{6}(2 \cdot a b c+b a c+a c b-b c a-c a b-2 \cdot c b a) \\
e_{3}^{(2)} & =\frac{1}{2}(a b c+c b a) \\
e_{3}^{(3)} & =\frac{1}{6}(a b c-b a c-a c b+b c a+c a b-c b a) .
\end{aligned}
$$

For the purposes of this example, we will apply $\partial[b]$ to each of these. Doing so, we get

$$
\begin{aligned}
& \partial[b] e_{3}^{(1)}=\frac{1}{6}(-2 a c+a c+a c-c a-c a+2 c a)=0 \\
& \partial[b] e_{3}^{(2)}=\frac{1}{2}(-a c-c a)=-e_{2}^{(1)} \\
& \partial[b] e_{3}^{(3)}=\frac{1}{6}(-a c-a c-a c+c a+c a+c a)=-e_{2}^{(2)} .
\end{aligned}
$$

Part 2 of Theorem 2.1 is needed later to show that the complex of injective words has a Hodge decomposition.

Theorem 2.1. Fix $n$ and $i \in\{1,2, \ldots, n\}$. Then:

(1) $\partial[i] \lambda_{n}^{(k)}=(-1)^{i-1} k \lambda_{n-1}^{(k)}$,

(2) $\partial[i] e_{n}^{(k)}=(-1)^{i-1} e_{n-1}^{(k-1)}$.

Before proving this theorem, let us verify two lemmas to be used in its proof.

Lemma 2.1. Among the $n$ places the letter $i$ could be inserted into a permutation $\sigma \in S_{[n] \backslash\{i\}}$ which has $j$ descents, $j+1$ choices yield permutations with $j$ descents, while the other $n-j-1$ choices all yield permutations with $j+1$ descents.

Proof. First we consider the case $i=n$, then use a graph for a permutation to generalize to all $i$. Notice that inserting $n$ between two letters descending letters preserves the number of descents, while inserting $n$ between two ascending letters increases the number of descents. Thus, there are $n-2-j$ ways to increase the number of descents by one by inserting $n$ between two ascending letters. In addition, inserting $n$ before the first letter gives one more way to increase the number of descents by one.

For $i \neq n$, the analysis will also need to consider whether or not $i$ is intermediate in value to the pair of consecutive labels where it is to be inserted. To this end, we define the graph of a permutation as follows:

Definition 2.1. For each $\pi \in S_{[n] \backslash\{i\}}$, define the related function $\pi^{\prime}$ : $[0, n] \rightarrow[0, n+1]$ by $\pi^{\prime}(j)=\pi(j)$ for $1 \leq j<i$ and $\pi^{\prime}(j)=\pi(j+1)$ for $n-1 \geq j>i$. In addition, let $\pi^{\prime}(0)=n+1$ and $\pi^{\prime}(n)=0$. Then the 
graph of $\pi$ is obtained by plotting the points $\left(j, \pi^{\prime}(j)\right)$ for each $j \in[0, n]$, and for each $j \in[0, n-1]$ connecting the point $\left(j, \pi^{\prime}(j)\right)$ to $\left(j+1, \pi^{\prime}(j+1)\right)$ by a straight line segment.

This graph has negative slope at each descent and positive slope at each ascent. Furthermore, it crosses the line $y=i$ with negative slope one more time than it does with positive slope, because it represents a continuous function which begins above the line $y=i$ and ends below the line $y=i$ (and which has nonzero slope everywhere it touches the line $y=i$ ).

We claim that the number of places to insert $i$ which will increase the number of descents by one is equal to sum of the number of ascents that do not cross the line together with the number of descents which do cross the line. This is clear except at the endpoints. By letting $\pi^{\prime}(0)=n+1$ and $\pi^{\prime}(n+1)=0$, we created descents at the initial and final positions in $\pi$, which are only counted above when the graph crosses the line $y=i$ at these points, namely when $\pi(1)<i$ and when $\pi(n)>i$, respectively. These are exactly the situations where inserting $i$ at the initial or final positions will indeed increase the number of descents by one.

We already observed that the number of descents crossing the line is one more than the number of ascents crossing the line. Thus, the total number of ways to increase the number of descents by one is one more than the total number of ascents, i.e., it is $n-j-1$, as desired. A similar argument shows that all of the remaining $j+1$ options will preserve the number of descents.

Lemma 2.2. If $\tau \in S_{n}$ is obtained from $\sigma \in S_{n \backslash\{i\}}$ by inserting $i$ after the $(d-1)$-st letter of $\sigma$, then $\operatorname{sgn}(\tau)=(-1)^{i-d} \operatorname{sgn}(\sigma)$.

Proof. If $d=1$, so $i=\tau_{1}$, then inserting $i$ created $i-1$ new descents, because the values $1, \ldots, i-1$ all appear later than the value $i$. Thus, $\operatorname{sgn}(\tau)=(-1)^{i-1} \operatorname{sgn}(\sigma)$ in this case, as desired. Now we proceed by induction on $d$. Moving the letter $i$ from position $r$ to position $r+1$ in $\tau$ by an adjacent transposition reverses the sign of $\tau$. Likewise, increasing $d$ from $r$ to $r+1$ reverses the sign of $(-1)^{i-d} \operatorname{sgn}(\sigma)$ from $(-1)^{i-r} \operatorname{sgn}(\sigma)$ to $(-1)^{i-(r+1)} \operatorname{sgn}(\sigma)$, so $\operatorname{sgn}(\tau)$ continues to agree with $(-1)^{i-1} \operatorname{sgn}(\sigma)$ as $d$ increases.

Proof of Theorem 2.1. We first prove identity (1). Note that

$$
\lambda_{n}^{(k)}=\sum_{i=0}^{k-1}(-1)^{i}\left(\begin{array}{c}
n+i \\
i
\end{array}\right)(-1)^{k-i-1} \sum_{\sigma} \operatorname{sgn}(\sigma) \sigma
$$


where the sum is over $\sigma$ with $k-i-1$ descents. Replacing $k-i-1$ by $j$ yields

$$
\lambda_{n}^{(k)}=\sum_{j=0}^{k-1}\left(\begin{array}{c}
n+k-j-1 \\
k-j-1
\end{array}\right)(-1)^{j} \sum_{\sigma} \operatorname{sgn}(\sigma) \sigma
$$

which simplifies to

$$
\lambda_{n}^{(k)}=(-1)^{k-1} \sum_{j=0}^{k-1}\left(\begin{array}{c}
n+k-j-1 \\
n
\end{array}\right) \sum_{\sigma} \operatorname{sgn}(\sigma) \sigma .
$$

In each of the last two equations, the sum is over $\sigma$ with $j$ descents. Similarly,

$$
\lambda_{n-1}^{(k)}=(-1)^{k-1} \sum_{j=0}^{k-1}\left(\begin{array}{c}
n+k-j-2 \\
n-1
\end{array}\right) \sum_{\sigma} \operatorname{sgn}(\sigma) \sigma .
$$

Lemma 2.1 shows that for each $\sigma \in S_{n \backslash\{i\}}$ with $j$ descents, there are $j+1$ permutations $\tau \in S_{n}$ with $j$ descents such that $\partial[i] \tau= \pm \sigma$ and there are $n-j-1$ permutations $\tau \in S_{n}$ with $j+1$ descents such that $\partial[i] \tau= \pm \sigma$. When our boundary operator $\partial[i]$ deletes $\tau_{d}$ from $\tau$ to obtain $\sigma \in S_{n \backslash\{i\}}$, we have $\partial[i] \tau=(-1)^{d-1} \sigma$, but Lemma 2.2 proves that in this case, $\operatorname{sgn}(\tau)=$ $(-1)^{i-d} \operatorname{sgn}(\sigma)$. Combining these signs, observe that

$$
\partial[i] \operatorname{sgn}(\tau) \tau=(-1)^{i-1} \operatorname{sgn}(\sigma) \sigma,
$$

independent of $d$.

Hence, the coefficient of $\sigma$ in $\partial[i] \lambda_{n}^{(k)}$ will be:

$$
\begin{aligned}
(-1)^{k-1} \cdot(-1)^{i-1} \operatorname{sgn}(\sigma)\left(\left(\begin{array}{c}
n+k-j-1 \\
n
\end{array}\right) \cdot(j+1)\right. & \\
& \left.\quad+\left(\begin{array}{c}
n+k-j-2 \\
n
\end{array}\right) \cdot(n-j-1)\right)
\end{aligned}
$$

which is equal to

$$
\begin{aligned}
(-1)^{k+i-2} \operatorname{sgn}(\sigma)\left(\begin{array}{c}
n+k-j-2 \\
n
\end{array}\right)\left(\begin{array}{r}
\frac{n+k-j-1}{k-j-1} \cdot(j+1) \\
+\frac{k-j-1}{k-j-1} \cdot(n-j-1)
\end{array}\right) .
\end{aligned}
$$

This simplifies to

$$
\begin{aligned}
(-1)^{k+i-2} \operatorname{sgn}(\sigma)\left(\begin{array}{c}
n+k-j-2 \\
n
\end{array}\right) & \cdot \frac{k n}{k-j-1} \\
& =(-1)^{k+i-2} \operatorname{sgn}(\sigma)\left(\begin{array}{c}
n+k-j-2 \\
n-1
\end{array}\right) \cdot k .
\end{aligned}
$$


This latter expression is the coefficient of $\sigma$ in $\lambda_{n-1}^{(k)}$ multiplied by $(-1)^{i-1} \cdot k$. This holds for each $\sigma$, regardless of the number of descents in $\sigma$, so we get

$$
\partial[i] \cdot \lambda_{n}^{(k)}=(-1)^{i-1} \cdot k \cdot \lambda_{n-1}^{(k)},
$$

confirming identity (1).

We next prove that identity (1) implies identity (2). Applying $\partial[i]$ to both sides of (2.3) gives:

$$
(-1)^{k-1}(-1)^{i-1} \cdot k \cdot \lambda_{n-1}^{(k)}=\partial[i] \sum_{j=1}^{n} k^{j} e_{n}^{(j)} .
$$

Applying (2.3) again, to the left-hand side yields,

$$
(-1)^{i+k-2} \cdot k \cdot(-1)^{k-1} \sum_{j=1}^{n-1} k^{j} e_{n-1}^{(j)}=\sum_{j=1}^{n} k^{j} \partial[i] e_{n}^{(j)} .
$$

Hence,

$$
(-1)^{i-1} \sum_{j=1}^{n-1} k^{j+1} e_{n-1}^{(j)}=\sum_{j=1}^{n} k^{j} \partial[i] e_{n}^{(j)} .
$$

So,

$$
0=k \partial[i] e_{n}^{(1)}+\sum_{j=2}^{n} k^{j}\left(\partial[i] e_{n}^{(j)}-(-1)^{i-1} e_{n-1}^{(j-1)}\right) .
$$

The fact that this holds for all $k$ implies that each coefficient of the polynomial in $k$ is 0 , and so we get $\partial[i] e_{n}^{(1)}=0$ and $\partial[i] e_{n}^{(j)}=(-1)^{i-1} e_{n-1}^{(j-1)}$.

The second statement in Theorem 2.1 is particularly interesting when compared to a result that appears in the work of Gerstenhaber and Schack [GS]. In that work, the authors show that for the boundary $\delta$ in the usual complex for computing Hochschild homology of a commutative algebra,

$$
\delta e_{n}^{(k)}=e_{n-1}^{(k)} \delta
$$

for all $n$ and $k$. Note that this bears some similarity to the result we prove in Theorem 2.1 for the simplicial case although in the simplicial case the boundary is applied on only one side and the Hodge index decreases by one rather than being constant.

For each $S \subseteq \underline{n}$, let $V_{S}$ denote the span of the $v_{i}$ for $i \in S$, and let $M_{S}$ denote the multilinear part of $V_{S}^{\otimes|S|}$. We will continue using the ReinerWebb point of view so that the injective words on the set $S$ form a basis for $M_{S}$. Note that $\partial\left(M_{S}\right) \subseteq \bigoplus_{i \in S} M_{S \backslash\{i\}}$, which means we can decompose $\partial$ as a sum of the operators $\partial[i]$ for $i \in S$.

Suppose $|S|=r$. Then the symmetric group $S_{r}$ acts on $M_{S}$ by permuting the positions in which letters appear in the injective words on $S$. For each $k$ with $1 \leq k \leq r$, let $M_{S}^{(k)}$ denote the image of $e_{r}^{(k)}$ under this action. By 
Theorem $2.1(2), \partial[i]\left(M_{S}^{(k)}\right) \subseteq M_{S \backslash\{i\}}$ for all $i \in S$. So if we let $M_{r}^{(k)}$ denote $\bigoplus_{|S|=r} M_{S}^{(k)}$, then

$$
\partial\left(M_{r}^{(k)}\right) \subseteq M_{r-1}^{(k-1)} .
$$

Thus, the complex $\left(M_{*}, \partial_{*}\right)$ splits as a direct sum of the sub-complexes $C^{(k)}$ where $C^{(k)}$ is

$$
0 \rightarrow M_{n}^{(k)} \rightarrow M_{n-1}^{(k-1)} \rightarrow \cdots \rightarrow M_{n-k+1}^{(1)} \rightarrow 0 .
$$

Let $H_{*}^{(k)}(M)$ denote the homology of the subcomplex $C^{(k)}$. We recall that $H_{r}^{(k)}(M)=0$ unless $r=n$.

Notice that the $S_{n}$-action on values which gives rise to the $S_{n}$-module structure studied in $[\mathbf{R W}]$ commutes with the $S_{r}$ action on positions in injective words in $M_{r}$. Thus, it makes sense to study the $S_{n}$-module structure of $M_{r}^{(k)}$ for each $r$ and for $H_{n}^{(k)}(M)$, with $S_{n}$ acting on values, despite the fact that the Eulerian idempotents act on positions. Our next result determines $H_{n}^{(k)}(M)$ as an $S_{n}$-module. To state this result, we will need some notation and results from $[\mathbf{H a}]$.

For each $\sigma \in S_{n}$, let $Z_{\sigma}$ denote the centralizer of $\sigma$. In [Ha], a character $\chi_{\sigma}$ is defined as the induction of a linear character $\Psi_{\sigma}$ from $Z_{\sigma}$ to $S_{n}$. To describe $\Psi_{\sigma}$, we first need a description of the $Z_{\sigma}$. Suppose $\sigma$ consists of $m_{\ell}$ $\ell$-cycles for each $\ell$. Then $Z_{\sigma}$ is the direct product of $C_{\ell} w r S_{m_{\ell}}$ where $C_{\ell}$ is the cyclic group of order $\ell$ and $w r$ denotes wreath product.

Let $\tau=\prod_{\ell}\left(\alpha_{\ell} ; \beta_{1}, \beta_{2}, \ldots, \beta_{m_{\ell}}\right)$ be an element of $Z_{\sigma}$ where $\alpha_{\ell} \in S_{m_{\ell}}$ and each $\beta_{i}$ is in $C_{\ell}$. Then

$$
\Psi_{\sigma}(\tau)=\prod_{\ell} \prod_{i=1}^{m_{\ell}} \gamma_{\ell}\left(\beta_{i}\right)
$$

where $\gamma_{\ell}$ is the linear character on $C_{\ell}$ which assigns $e^{2 \pi i / \ell}$ to the generator of $C_{\ell}$.

The following theorem from [Ha] will help us understand $M_{r}^{(k)}$ :

Theorem 2.2 (Hanlon). For each $n$ and $k$, let $I^{(k)}$ denote the left ideal in $\mathrm{C} S_{n}$ generated by $e_{n}^{(k)}$. As an $S_{n}$-module,

$$
\operatorname{sgn} * I_{n}^{(k)}=\bigoplus_{\sigma} \chi_{\sigma}
$$

where the sum is over a choice of representative from each conjugacy class that consists of permutations with exactly $k$ cycles, and $*$ denotes internal product.

The $S_{n}$-modules $\bigoplus_{\sigma} \chi_{\sigma}$ in Theorem 2.2 have also appeared in a completely different context, in work of Bergeron, Bergeron and Garsia on the free Lie algebra $[\mathbf{B B G}]$. In contrast to Theorem 2.2 and $[\mathbf{B B G}]$, we will 
study $S_{n}$-modules in which we sum over conjugacy classes of derangements rather than conjugacy classes of permutations.

Our next result determines each $H_{n}^{(k)}(M)$ as an $S_{n}$-module, thereby providing a refinement of the theorem of Reiner and Webb which gives the $S_{n}$-module structure of $H_{n}(M)$.

Theorem 2.3. For each $n$ and $k$,

$$
\operatorname{sgn} * H_{n}^{(k)}(M)=\bigoplus_{\sigma} \chi_{\sigma}
$$

where $*$ denotes the internal product, and the sum is over a choice of representative from each conjugacy class consisting of derangements with exactly $k$ cycles. In particular, $\operatorname{dim}\left(H_{n}^{(k)}(M)\right)$ equals the number of derangements with exactly $k$ cycles.

We will use cycle indices to prove Theorem 2.3. For each $\sigma \in S_{n}$, let $j_{i}(\sigma)$ denote the number of $i$-cycles of $\sigma$. Let $a_{1}, a_{2}, \ldots$ be a set of commuting indeterminates. Define $Z(\sigma)$, the cycle indicator of $\sigma$, to be

$$
Z(\sigma)=a_{1}^{j_{1}(\sigma)} a_{2}^{j_{2}(\sigma)} a_{3}^{j_{3}(\sigma)} \ldots
$$

So $Z(\sigma)$ is a monomial which identifies the cycle type of $\sigma$. Thus, $\sigma$ and $\tau$ are conjugate in $S_{n}$ iff $Z(\sigma)=Z(\tau)$.

Let $\Psi$ be a class function on $\mathbf{C} S_{n}$. The cycle index of $\Psi$ is

$$
Z(\Psi)=\frac{1}{n !} \sum_{\sigma \in S_{n}} \Psi(\sigma) Z(\sigma) .
$$

Since the monomial $Z(\sigma)$ uniquely identifies the conjugacy class of $\sigma$, two class functions are identical if and only if they have the same cycle index.

We will need two results about cycle indices from $[\mathbf{H a}]$. In the results below, $\varepsilon_{t}$ denotes the trivial representation of $S_{t}\left(\right.$ so $Z\left(\varepsilon_{t}\right)=\frac{1}{t !} \sum_{\sigma \in S_{t}} Z(\sigma)$ ) and [] denotes the composition product on $\mathbf{C}\left[\left[a_{1}, a_{2}, \ldots\right]\right]^{*}$, i.e., for $A, B \in$ $\mathbf{C}\left[\left[a_{1}, a_{2}, \ldots\right]\right]$,

$$
A[B]=A\left(a_{i} \leftarrow B\left(a_{j} \leftarrow a_{i j}\right)\right)
$$

where $\leftarrow$ denotes substitution. Recall that $\mu$ denotes the ordinary number theoretic Möbius function.

The following two results are proved in $[\mathbf{H a}]$ :

Theorem 2.4 (Hanlon). Let $\sigma \in S_{n}$ with $Z(\sigma)=a_{1}^{j_{1}} a_{2}^{j_{2}} \ldots a_{n}^{j_{n}}$. Then

$$
Z\left(\chi_{\sigma}\right)=\prod_{\ell=1}^{n} Z\left(\varepsilon_{j_{\ell}}\right)\left[\frac{1}{\ell} \sum_{d \mid \ell} \mu(d) a_{d}^{\ell / d}\right] .
$$


Theorem 2.5 (Hanlon). Let $I_{n}^{(k)}$ denote the left ideal in $\mathbf{C} S_{n}$ generated by $e_{n}^{(k)}$. Then

$$
\sum_{n, k} Z\left(I_{n}^{(k)}\right) \lambda^{k}=\prod_{\ell}\left(1+(-1)^{\ell} a_{\ell}\right)^{\frac{-1}{\ell} \sum_{d \mid \ell} \mu(d) \lambda^{\ell / d}}
$$

We are now ready to give:

Proof of Theorem 2.3. Recall that the Euler characteristic of a chain complex is the alternating sum of the ranks of its homology groups, and the Hopf Trace Formula refines this to a statement about module structure. Since the homology of $\left(M_{*}, \partial_{r}\right)$ vanishes except at the top degree, we deduce that

$$
Z\left(H_{n}^{(k)}(M)\right)=\sum_{r=n-k+1}^{n} Z\left(M_{r}^{(k-(n-r))}\right)(-1)^{n-r} .
$$

Note that

$$
M_{r}=\bigoplus_{|S|=r} M_{S}=\operatorname{ind}{ }_{S_{r} \times S_{n-r}}^{S_{n}}\left(\operatorname{Reg}_{r} \otimes \varepsilon_{n-r}\right)
$$

where $\operatorname{Reg}_{r}$ denotes the right-regular representation of $S_{r}$. It follows that

$$
M_{r}^{k-n+r}=\operatorname{ind} \underset{S_{r} \times S_{n-r}}{S_{n}}\left(I_{r}^{(k-n+r)} \otimes \varepsilon_{n-r}\right) .
$$

We will use one other well-known fact about cycle indices, namely that for any virtual characters $\Psi$ of $S_{r}$ and $\Theta$ of $S_{n-r}$,

$$
Z\left(\operatorname{ind} S_{S_{r} \times S_{n-r}}^{S_{n}}(\Psi \otimes \Theta)\right)=Z(\Psi) Z(\Theta) .
$$

Combining these facts we have:

$$
\begin{aligned}
& \sum_{n} \sum_{k=1}^{n} Z\left(H_{n}^{(k)}(M)\right) \lambda^{k} \\
& =\sum_{n} \sum_{k=1}^{n} \sum_{r=n-k+1}^{n} Z\left(M_{r}^{(k-(n-r))}\right)(-1)^{n-r} \lambda^{k} \\
& =\sum_{n} \sum_{k=1}^{n} \sum_{r=n-k+1}^{n} Z\left(I_{r}^{(k-n+r)}\right)\left(\lambda^{k-n+r}\right)\left(-\lambda^{n-r}\right) Z\left(\varepsilon_{n-r}\right) \\
& =\left(\sum_{r, t} Z\left(I_{r}^{(t)}\right) \lambda^{t}\right) \cdot\left(\sum_{m=0}^{\infty}(-\lambda)^{m} Z\left(\varepsilon_{m}\right)\right) \\
& =\left(\prod_{\ell}\left(1+(-1)^{\ell} a_{\ell}\right)^{\frac{-1}{\ell} \sum_{d \mid \ell} \mu(d) \lambda^{\ell / d}}\right) \cdot \exp \left(\sum_{p} \frac{(-\lambda)^{p} a_{p}}{p}\right)
\end{aligned}
$$


in the last step using the well-known fact that

$$
\sum_{m} Z\left(\varepsilon_{m}\right)=\exp \left(\sum_{i} \frac{a_{i}}{i}\right) .
$$

Thus,

$$
\sum_{n} \sum_{k=1}^{n} Z\left(\operatorname{sgn} * H_{n}^{(k)}(M)\right) \lambda^{k}=F_{1} \cdot F_{2}
$$

where

$$
F_{1}=\prod_{\ell}\left(1-a_{\ell}\right)^{\frac{-1}{\ell} \sum_{d \mid \ell} \mu(d) \lambda^{\ell / d}}
$$

and

$$
F_{2}=\exp \left(-\sum_{p} \frac{\lambda^{p} a_{p}}{p}\right) .
$$

We can rewrite $F_{1}$ as

$$
\begin{aligned}
F_{1} & =\prod_{\ell} \exp \left(\ln \left(1-a_{\ell}\right)\left(\frac{-1}{\ell} \sum_{d \mid \ell} \mu(d) \lambda^{\ell / d}\right)\right) \\
& =\prod_{\ell} \exp \left(\sum_{m=1}^{\infty} \frac{a_{\ell}^{m}}{m \ell} \sum_{d \mid \ell} \mu(d) \lambda^{\ell / d}\right) .
\end{aligned}
$$

Letting $p$ denote $\ell / d$ and $n$ denote $m d$, we have

$$
\begin{aligned}
F_{1} & =\exp \left(\sum_{p, d, m} \frac{1}{m p d} \mu(d) \lambda^{p} a_{d p}^{m}\right) \\
& =\exp \left(\sum_{p} \frac{\lambda^{p} a_{p}}{p}\right)\left[\sum_{d, m} \frac{1}{m d} \mu(d) a_{d}^{m}\right] \\
& =\exp \left(\sum_{p} \frac{\lambda^{p} a_{p}}{p}\right)\left[\sum_{n} \frac{1}{n} \sum_{d \mid n} \mu(d) a_{d}^{n / d}\right] \\
& =\exp \left(\sum_{p} \frac{\lambda^{p} a_{p}}{p}\right) \exp \left(\sum_{p} \frac{\lambda^{p} a_{p}}{p}\right)\left[\sum_{\ell \geq 2} \frac{1}{\ell} \sum_{d \mid \ell} \mu(d) a_{d}^{\ell / d}\right] .
\end{aligned}
$$

So,

$$
\sum_{n, k} Z\left(\operatorname{sgn} * H_{n}^{(k)}(M)\right) \lambda^{k}=\exp \left(\sum_{p} \frac{\lambda^{p} a_{p}}{p}\right)\left[\sum_{\ell \geq 2} \frac{1}{\ell} \sum_{d \mid \ell} \mu(d) a_{d}^{\ell / d}\right],
$$

which proves the result. 
We conclude this section by showing how to recover Theorem 1.1 from Theorem 2.3. Setting $\lambda=1$ in Theorem 2.3, we obtain

$$
\begin{aligned}
\sum_{n} Z\left(\operatorname{sgn} * H_{n}(M)\right) & =\left(\sum_{n, k} Z\left(\operatorname{sgn} * H_{n}^{(k)}(M)\right)\right) \\
& =\exp \left(\sum_{p} \frac{a_{p}}{p}\right)\left[\sum_{\ell \geq 2} \frac{1}{\ell} \sum_{d \mid \ell} \mu(d) a_{d}^{\ell / d}\right] \\
& =\exp \left(\sum_{p} \sum_{\ell \geq 2} \sum_{d \mid \ell} \frac{1}{p \ell} \mu(d) a_{d p}^{\ell / d}\right) .
\end{aligned}
$$

Letting $u=\ell / d$, we have

$$
\sum_{n} Z\left(\operatorname{sgn} * H_{n}(M)\right)=\exp \left(\sum_{p, d, u} \frac{1}{p d u} \mu(d) a_{d p}^{u}\right) \cdot \exp \left(-\sum_{p} \frac{a_{p}}{p}\right)
$$

where the latter factor accounts for the provision that $\ell$ cannot equal 1 . So, substituting $v$ for $p d$ yields

$$
\begin{aligned}
\sum_{n} Z\left(\operatorname{sgn} * H_{n}(M)\right) & =\exp \left(\sum_{u, v} \frac{a_{v}^{u}}{u v}\left(\sum_{d \mid v} \mu(d)\right)\right) \cdot \exp \left(-\sum_{p} \frac{a_{p}}{p}\right) \\
& =\exp \left(\sum_{u} \frac{a_{1}^{u}}{u}\right) \cdot \exp \left(-\sum_{p} \frac{a_{p}}{p}\right) .
\end{aligned}
$$

Thus,

$$
\begin{aligned}
\sum_{n} Z\left(H_{n}(M)\right) & =\left(\frac{1}{1-a_{1}}\right) \cdot \exp \left(\sum_{p} \frac{(-1)^{p} a_{p}}{p}\right) \\
& =\left(\sum_{k} Z\left(\operatorname{Reg}_{k}\right)\right) \cdot\left(\sum_{m=0}^{\infty}(-1)^{m} Z\left(\varepsilon_{m}\right)\right) \\
& =\sum_{n} \sum_{k} Z\left(\operatorname{ind}_{S_{n-k} \times S_{k}}^{S_{n}}\left(\varepsilon_{n-k} \otimes \operatorname{Reg}_{k}\right)\right)(-1)^{n-k}
\end{aligned}
$$

which is the Reiner-Webb Theorem.

\section{Signed random to random shuffles.}

In recent work, Uyemura-Reyes $[\mathbf{U y}$ ] considers random to random shuffling of a deck of $n$ cards and conjectures that the transition matrix, when normalized to have integer entries, also has integer spectrum. For small values of $n$, he notes that the nullspace of the transition matrix has dimension equal to 
the number of derangements of $n$ and that the nullspace, as an $S_{n}$-module, carries the same representation, up to a sign twist, as the representation that appears on the right-hand side of Theorem 1.1. In this section, we explain this phenomenon by studying the Laplacian $L$ of the complex $\left(M_{*}, \partial_{*}\right)$. We show that if the normalized random-to-random shuffle operator has integral spectrum (as conjectured in $[\mathbf{U} \mathbf{y}]$ ), then the Laplacian on each chain group in the complex of injective words will also have integral spectrum.

Definition 3.1. For each $r$, let $v_{r}$ and $\Upsilon_{r}$ be the elements of the group algebra $\mathbf{C} S_{r}$ given by:

$$
v_{r}=r \cdot i d+\sum_{u<v}(v, u, u+1, \ldots, v-1)+\sum_{u>v}(v, u, u-1, \ldots, v+1)
$$

and

$$
\begin{aligned}
\Upsilon_{r}= & r \cdot i d+\sum_{u<v}(-1)^{v-u}(v, u, u+1, \ldots, v-1) \\
& +\sum_{u>v}(-1)^{u-v}(v, u, u-1, \ldots, v+1) .
\end{aligned}
$$

If we think of $S_{r}$ as acting on a deck of $r$ cards by permuting the positions of the cards, then $v_{r}$ sums permutations which pick at random two positions $v$ and $u$ and move the card in position $v$ to position $u$. Thus $v_{r}$ is $r^{2}$ times the transition matrix for random to random shuffling. Note that $\Upsilon_{r}$ is simply $v_{r}$ twisted by the sign. Thus we will refer to $\Upsilon_{r}$ as the signed random to random shuffle element in $\mathbf{C} S_{r}$.

The following conjecture appears in the dissertation of Uyemura-Reyes:

Conjecture 3.1 (Uyemura-Reyes). The eigenvalues of $v_{n}$ are (rational) integers.

As in Section 2, we will use the collection $B_{r}$ of injective words of length $r$ on the alphabet $\{1,2, \ldots, n\}$ as a basis for $M_{r}$. Let $\delta_{r}: M_{r} \rightarrow M_{r+1}$ be the transpose of $\partial_{r+1}$ with respect to the inner products on $M_{r}$ and $M_{r+1}$ which have $B_{r}$ and $B_{r+1}$ as orthonormal bases. So if $D$ is the matrix for $\partial_{r+1}$ with respect to the bases $B_{r}$ and $B_{r+1}$, then $D^{t}$ is the matrix for $\delta_{r}$ with respect to the same bases. Note that $\delta_{*}$ is a coboundary on $M_{*}$. We let $H^{*}(M)$ denote the cohomology with respect to this coboundary.

Let $\Lambda_{r}: M_{r} \rightarrow M_{r}$ be the Laplacian

$$
\Lambda_{r}=\delta_{r-1} \cdot \partial_{r}+\partial_{r+1} \cdot \delta_{r} .
$$

We recall the well-known fact that a basis for the kernel of the Laplacian $\Lambda_{r}$ gives a simultaneous basis for $H_{r}(M)$ and $H^{r}(M)$.

Theorem 3.1. The Laplacian on the top-dimensional chain group satisfies $\Lambda_{n}=\Upsilon_{n}$. 
Proof. To apply the coboundary $\delta_{n-1}$ to a basis element $j_{1} j_{2} \ldots j_{n-1}$, we must sum over all sequences $i_{1} i_{2} \ldots i_{n}$ with coefficient being the $j, \underline{i}$ entry from $\partial_{n}$. Since $\partial_{n}\left(i_{1} \ldots i_{n}\right)$ is a sum of terms of the form $\pm j_{1} \ldots j_{n-1}$ where $j_{1} \ldots j_{n-1}$ is obtained by deleting an entry from $i_{1} \ldots i_{n}$, the $\underline{j}, \underline{i}$ entry of $\partial_{n}$ is 0 unless $\underline{j}$ is a subsequence of $\underline{i}$. It follows that if $v$ is the single number in $\{1,2, \ldots, n\}$ which is missing from $\left\{j_{1}, \ldots, j_{n-1}\right\}$ then

$$
\delta_{n-1}\left(j_{1} \ldots j_{n-1}\right)=\left(v j_{1} \ldots j_{n-1}\right)-\left(j_{1} v j_{2} \ldots j_{n-1}\right)+\left(j_{1} j_{2} v j_{3} \ldots j_{n-1}\right)-\ldots
$$

So $\delta_{n-1} \partial_{n}$ is the operator which acts on a sequence $i_{1} i_{2} \ldots i_{n}$ by removing an element and re-inserting it in all possible ways. Moreover, if the removed element occupies position $u$ and it is re-inserted in position $v$ then the sign of that operation is $(-1)^{(u-1)+(v-1)}=(-1)^{v-u}$. On the other hand, $\partial_{n+1} \delta_{n}=$ 0 . It follows that $\Lambda_{n}$ is equal to $\Upsilon_{n}$ which proves the result.

As noted above, Uyemura-Reyes conjectures that the spectrum of $v_{n}$ is integral from which it would follow that the spectrum of $\Lambda_{n}$ is integral. We end this section by relating $\Lambda_{r}$ to $\Lambda_{n}$. From this relationship one can deduce that if Conjecture 3.1 holds, then $\Lambda_{r}$ has integral spectrum for all $r$.

Theorem 3.2. Let $i_{1} \ldots i_{r}$ be a basis element of $M_{r}$. Let $A$ denote $\left\{i_{1}, \ldots\right.$, $\left.i_{r}\right\}$ and let $\bar{A}$ denote the complement of $A$ in $\{1,2, \ldots, n\}$. Then,

$$
\Lambda_{r}\left(i_{1} \ldots i_{r}\right)=\left((r+1)(n-r) I+\Upsilon_{r}+\sum_{a \in A, b \in \bar{A}}(a, b)\right)\left(i_{1} \ldots i_{r}\right)
$$

where $\Upsilon_{r}$ is acting by permutation of positions on $i_{1} \ldots i_{r}$ whereas $(a, b)$ in the last summation is acting by permuting the values of the $i_{j}$ within the set $\{1,2, \ldots, n\}$.

Proof. We write $\Lambda_{r}\left(i_{1} \ldots i_{r}\right)$ as a sum of three expressions

$$
\Lambda_{r}\left(i_{1} \ldots i_{r}\right)=X+Y+Z
$$

where $\mathrm{X}$ is the sum of all terms in $\partial_{r+1} \delta_{r}\left(i_{1} \ldots i_{r}\right)$ in which $\delta_{r}$ inserts an element $j$ of $\bar{A}$ in some position $u$ and then $\partial_{r+1}$ removes the same number $j$, where $\mathrm{Y}$ is the sum of all terms in $\delta_{r-1} \partial_{r}\left(i_{1} \ldots i_{r}\right)$ in which $\partial_{r}$ removes an element $j \in A$ and $\delta_{r-1}$ re-inserts that same element $j$ and where $\mathrm{Z}$ is the remaining terms in $\Lambda_{r}\left(i_{1} \ldots i_{r}\right)$.

Note that:

$$
X=(r+1)(n-r)
$$

and that:

$$
Y=\Upsilon_{r}
$$

It will take some considerable effort now to analyze $Z$.

The terms $\tau$ in $\partial_{r+1} \delta_{r}\left(i_{1} \ldots i_{r}\right)$ that contribute to $Z$ are those in which an element $j$ from $\bar{A}$ is inserted into $i_{1} \ldots i_{r}$ at some position $u$ by $\delta_{r}$ and then one of the $i_{\ell}$ is removed by $\partial_{r+1}$. For each such $\tau$, there is a corresponding 
term $\hat{\tau}$ in $\delta_{r-1} \partial_{r}\left(i_{1} \ldots i_{r}\right)$ where $i_{\ell}$ is removed first by $\partial_{r}$ and then $j$ is inserted in position corresponding to $u$ by $\delta_{r-1}$. It is straightforward to check that $\tau=-\hat{\tau}$ and so these terms cancel.

There is one circumstance in which this cancellation does not eliminate every term. These are the terms $\tau$ where $j$ is inserted immediately behind $i_{\ell}$, i.e., where $u=\ell+1$. In this case, the term $\hat{\tau}$ which should cancel $\tau$ is already committed to cancel the term $\tau^{\prime}$ in which $j$ is inserted immediately in front of $i_{\ell}$.

For $j \in \bar{A}$ and $i_{\ell} \in A$, the term in which $j$ is inserted immediately behind $i_{\ell}$ and then $i_{\ell}$ is deleted has sign +1 and is obtained by acting on $i_{1} i_{2} \ldots i_{r}$ with the transposition $\left(j, i_{\ell}\right) \in S_{n}$. The result follows.

It is worth noting that the operator $\Upsilon_{r}$, acting on positions, commutes with the action of $S_{n}$ on words of length $r$ in $1,2, \ldots, n$. The first part of Theorem 3.3 alternatively follows from $[\mathbf{F}]$ or from the shelling for $K_{n}$ in $[\mathrm{BW}]$.

Theorem 3.3. For $r<n$ :

(1) $\Lambda_{r}$ is positive definite.

(2) If Conjecture 3.1 holds, then the spectrum of $\Lambda_{r}$ is integral.

Proof. For this argument, it will be helpful to reconceptualize $M_{r}$. Let $i_{1} i_{2} \ldots i_{r}$ be a basis element of $M_{r}$ and let $\left\{j_{1}, \ldots, j_{n-r}\right\}=\bar{A}$. We will identify $i_{1} \ldots i_{r}$ with

$$
\left[i_{1} \ldots i_{r}\right]=\frac{1}{(n-r) !} \sum_{\sigma \in S_{n-r}} i_{1} \ldots i_{r} j_{\sigma 1} j_{\sigma 2} \ldots j_{\sigma(n-r)} \in M_{n} .
$$

The advantage this has is that the operator $\sum_{a \in A, b \in \bar{A}}(a, b)$ whose action seemed to depend on the actual set $A$ can be redefined as the operator:

$$
\Gamma=\sum_{a \in\{1, \ldots, r\}, b \in\{r+1, \ldots, n\}}(a, b)
$$

where the permutation $(a, b)$ is acting now by permutation of positions. So, $\Lambda_{r}=((r+1)(n-r) I)+\Upsilon_{r}+\Gamma$.

Let $\Omega=((r+1)(n-r) I)+\Gamma$. Note that $\Omega$ can be written as:

$$
\Omega=((r+1)(n-r) I)+T(1, n)-T(1, r)-T(r+1, n)
$$

where $T(u, v)=\sum_{u \leq a<b \leq v}(a, b)$. Recall that

$$
M_{r}=\left(\operatorname{Reg}_{r} \otimes \varepsilon_{n-r}\right) \uparrow_{S_{r} \times S_{n-r}}^{S_{n}}
$$

where $\operatorname{Reg}_{r}$ denotes the regular representation of $S_{r}$. Therefore,

$$
M_{r}=\bigoplus_{\alpha \vdash r} f_{\alpha}\left(S^{\alpha} \otimes \varepsilon_{n-r}\right) \uparrow{ }_{S_{r} \times S_{n-r}}^{S_{n}}
$$


where $S^{\alpha}$ denotes the Specht module indexed by $\alpha$ and $f_{\alpha}$ is the number of standard Young tableaux of shape $\alpha$.

For $x$ a square in row $i$ and column $j$ of a Ferrer's diagram of $\alpha$, recall that $c_{x}$, the content of $x$, is $j-i$. A well-known result from the representation theory of $S_{n}$ states that for a Specht module $S^{\lambda}$ with $\lambda \vdash n, T_{n}$ acts as the scalar $\sum_{x \in \lambda} c_{x}$. It follows that for every $\lambda \vdash n$ which occurs in $\left(S^{\alpha} \otimes\right.$ $\left.\varepsilon_{n-r}\right) \uparrow_{S_{r} \times S_{n-r}}^{S_{n}}$, the operator $\Omega$ acts as the scalar:

$$
(r+1)(n-r)+\sum_{x \in \lambda} c_{x}-\sum_{x \in \alpha} c_{x}-\sum_{x \in(n-r)} c_{x}
$$

which simplifies to expression (3.2):

$$
(r+1)(n-r)+\sum_{x \in \lambda / \alpha} c_{x}-\left(\begin{array}{c}
n-r \\
2
\end{array}\right)
$$

We will make two observations based on this formula. The first is that the eigenvalues of $\Omega$ are integral. Also, both $\Omega$ and $\Upsilon_{r}$ are easily seen to be diagonalizable. Moreover, they commute. It follows that the eigenvalues of $\Lambda_{r}=\Upsilon_{r}+\Omega$ can be written as sums of eigenvalues of $\Upsilon_{r}$ and $\Omega$. However, $\Upsilon_{r}$ is conjugate to $v_{r}$ and hence has the same spectrum. Thus, if Conjecture 3.1 holds, then all eigenvalues of $\Lambda_{r}$ are sums of integers. This proves Part (2) of the theorem.

To prove that $\Lambda_{r}$ is positive definite, first note that it is enough to show that $\Omega$ is positive definite since $\Upsilon_{r}$ is positive semi-definite, being a direct sum of $\left(\begin{array}{l}n \\ r\end{array}\right)$ copies of the Laplacian in top degree for the case with $n=r$. To see that $\Omega$ is positive definite, we start with the expression for the action of $\Omega$ on copies of $S_{n}$ irreducibles given in (3.2) above. The first observation follows from the fact that $S^{\lambda}$ has nonzero multiplicity in $\left(S^{\alpha} \otimes \varepsilon_{n-r}\right) \uparrow_{S_{r} \times S_{n-r}}^{S_{n}}$ if and only if $\lambda / \alpha$ is a horizontal strip. Let $\left(\rho_{1}, \gamma_{1}\right),\left(\rho_{2}, \gamma_{2}\right), \ldots,\left(\rho_{n-r}, \gamma_{n-r}\right)$ be the coordinates of the squares in $\lambda / \alpha$. The fact that $\lambda / \alpha$ is a horizontal strip implies that $1 \leq \gamma_{1}<\gamma_{2} \cdots<\gamma_{n-r}$. Thus,

$$
\sum_{s} \gamma_{s} \geq\left(\begin{array}{c}
n-r \\
2
\end{array}\right)+(n-r) .
$$

Also, observe that if a square $x$ of the Ferrer's diagram of $\lambda / \alpha$ is in row $i$, then there are $(i-1)$ squares of $\alpha$ in the rows above it. So, $\sum\left(\rho_{s}-1\right) \leq r$, i.e., $\sum \rho_{s} \leq r+(n-r)$.

Putting these bounds together gives that the eigenvalue $\omega$ given in formula (3.2) satisfies

$$
\omega \geq(r+1)(n-r)+\left(\begin{array}{c}
n-r \\
2
\end{array}\right)+(n-r)-(r+(n-r))-\left(\begin{array}{c}
n-r \\
2
\end{array}\right)
$$

which simplifies to:

$$
\omega \geq(r+1)(n-r)-r>0 .
$$




\section{References}

[BBG] F. Bergeron, N. Bergeron and A.M. Garsia, Idempotents for the free Lie algebra and q-enumeration, Invariant Theory and Tableaux (Minneapolis, MN, 1988), IMA Vol. Math. Appl., 19, Springer, New York, 1989, 166-190, MR 1035495, Zbl 0721.17006.

[BW] A. Björner and M. Wachs, On lexicographically shellable posets, Trans. Amer. Math. Soc., 277 (1983), 323-341, MR 0690055, Zbl 0514.05009.

[CHR] A.R. Calderbank, P. Hanlon and R.W. Robinson, Partitions into even and odd block size and some unusual characters of the symmetric groups, Proc. London Math. Soc. (3), 53 (1986), 288-320, MR 0850222, Zbl 0602.20017.

[F] F.D. Farmer, Cellular homology for posets, Math. Japan, 23 (1978/79), 607-613, MR 0529895, Zbl 0416.55003.

[FH] J. Friedman and P. Hanlon, On the Betti numbers of chessboard complexes, J. Algebraic Combinatorics, 8 (1998), 193-203, MR 1668521, Zbl 0914.55013.

[GS] M. Gerstenhaber and S.D. Schack, A Hodge-type decomposition for commutative algebra cohomology, J. Pure Appl. Algebra, 48 (1987), 229-247, MR 0917209, Zbl 0671.13007.

[Ha] P. Hanlon, The action of $S_{n}$ on the components of the Hodge decomposition of Hochschild homology, Michigan Math. J., 37 (1990), 105-124, MR 1042517, Zbl 0701.16010.

[Lo] J.-L. Loday, Partition Eulerienne et operations en homologie cyclique, C.R. Acad. Sci. Pari Ser. I Math., 307 (1988), 283-286, MR 0958781, Zbl 0669.13006.

[RW] V. Reiner and P. Webb, The combinatorics of the bar resolution in group cohomology, J. Pure Appl. Algebra (to appear).

[So] L. Solomon, A decomposition of the group algebra of a finite Coxeter group, J. Algebra, 9 (1968), 220-239, MR 0232868, Zbl 0186.04503.

[Uy] Jay-Calvin Uyemura-Reyes, Random Walk, Semi-Direct Products and Card Shuffling, Ph. D. Thesis, Stanford University, May 2002.

Received January 2, 2003 and revised April 22, 2003. The second author was supported by an NSF postdoctoral research fellowship.

Department of Mathematics

UNIVERSITY OF MiCHIGAN

Ann Arbor, Michigan 48109-1109

E-mail address: hanlon@umich.edu

Department of Mathematics

UNIVERSITY OF MICHIGAN

Ann Arbor, Michigan 48109-1109

E-mail address: plhersh@umich.edu 